\title{
Assessment of Contraceptive Counseling and Contraceptive Use in Women After Bariatric Surgery
}

\author{
Michiel A. Damhof ${ }^{1}$ (D) $\cdot$ Esther Pierik ${ }^{2} \cdot$ Lisanne L. Krens $^{3} \cdot$ Marloes Vermeer $^{4} \cdot$ Marc J. van Det $^{5} \cdot$ Eric N. van Roon ${ }^{2,6}$
}

Published online: 9 July 2019

(C) The Author(s) 2019

\begin{abstract}
Background Reproductive-aged women are, according to American and European guidelines, recommended to avoid pregnancy for 12-24 months after bariatric surgery. Oral contraceptives may have suboptimal efficacy after malabsorptive bariatric procedures.

Aim The aim of this study was to assess contraceptive use pre- and postoperatively in women who underwent bariatric surgery in two obesity clinics in The Netherlands. Also, the recall of contraceptive and pregnancy counseling was investigated.

Methods A validated questionnaire was performed among women aged 18-45 years who underwent bariatric surgery from October 2017 through August 2018.

Results In total, 230 women were eligible for final analysis. Postoperatively, $60 \%$ used safe contraception, $16.1 \%$ unsafe contraception, and $23.9 \%$ no contraception. In this study, $43.7 \%$ of women using a potential unsafe contraceptive method preoperatively switched to a safe method of contraception postoperatively $(p<0.0001)$. Only $62.6 \%$ of women confirmed to have received contraceptive counseling, mainly preoperatively. The odds ratio for receiving contraceptive counseling and using safe contraceptive methods compared with not receiving contraceptive counseling was 2.20 (95\% CI, 1.27-3.79; $p=0.005$ ). Eighty-three percent confirmed that they have received counseling regarding delaying a pregnancy, and $52.6 \%$ were familiar with the recommendation to avoid a pregnancy for 24 months postoperatively.

Conclusions In our study, $60 \%$ of women are using safe contraception postoperatively. Contraceptive counseling is suboptimal as $62.6 \%$ recall receiving counseling. Those who confirmed receiving counseling were more likely to use safe contraception after bariatric surgery. More counseling and monitoring in the postoperative and in the outpatient setting is recommended.
\end{abstract}

Keywords Bariatric surgery $\cdot$ Gastric bypass $\cdot$ Contraception $\cdot$ Counseling $\cdot$ Pregnancy

Michiel A. Damhof

m.damhof@zgt.nl

1 Department of Clinical Pharmacy, Ziekenhuisgroep Twente, Almelo, The Netherlands

2 Department of Pharmacotherapy, Epidemiology and Economics, Department of Pharmacy, University of Groningen, Groningen, The Netherlands

3 Department of Clinical Pharmacy, Maasstad Ziekenhuis, Rotterdam, The Netherlands

4 ZGT Academy, Ziekenhuisgroep Twente, Almelo, The Netherlands

5 Department of Surgery, Ziekenhuisgroep Twente, Almelo, The Netherlands

6 Department of Clinical Pharmacy and Clinical Pharmacology, Medical Centre Leeuwarden, Leeuwarden, The Netherlands

\section{Introduction}

Bariatric surgery is a safe and effective treatment for morbid obesity. Approximately 50\% of women undergoing bariatric surgery are of reproductive age [1-3]. The American College of Obstetricians and Gynecologists (ACOG) and the American Society of Metabolic and Bariatric Surgery (ASMBS) [4] recommend preventing pregnancy during the first 12-24 months following bariatric surgery [5-7]. The interval of 12-24 months is important since the initial months following surgery are associated with rapid weight loss, which could potentially cause adverse effects to a pregnancy, such as intrauterine growth restriction, anemia, and neural tube defects due to maternal nutritional status. Pregnancies occurring sooner than 24 months after bariatric surgery were associated with a higher incidence of preterm deliveries [6]. Furthermore, several international cohort studies reported an increased risk 
for significant maternal anemia and an increase in small-forgestational-age babies for women with prior bariatric surgery when compared with BMI-matched controls. In these studies, there was no difference in the incidence of congenital malformations between the two groups. On the other hand, these studies also showed a decreased risk for maternal gestational diabetes mellitus, a decreased incidence of preeclampsia, and a decreased incidence of emergent cesarean delivery for women after bariatric surgery compared with BMImatched controls [8-11].

The altered reproductive hormone profile associated with morbid obesity seems to reverse, either partially or totally, after bariatric surgery. This may result in restoration of the normal ovulation and enhanced fertility. The risk of an unplanned pregnancy may therefore be increased after bariatric surgery, and the use of adequate contraception for women who undergo bariatric surgery is important [12].

The European Society of Contraception (ESC) and Centers for Disease Control (CDC) medical eligibility criteria for contraceptive discourage the use of combined oral contraceptives (COCs) or progesterone-only pills (POPs) in postbariatric patients, due to possible decreased efficacy secondary to malabsorptive procedures [13, 14]; however, adequate evidence is limited to a few pharmacokinetic studies.

Andersen et al. found no significant differences in basal levels of estradiol, estrone, testosterone, and progesterone compared with non-operated obese patients [15]. Victor et al. demonstrated significantly lower plasma levels of both norethisterone and levonorgestrel, suggesting a reduced absorptive capacity and risk for contraceptive failure [16]. Both studies, however, were performed in women who had undergone jejunoileal bypass, a procedure that is no longer performed.

Recently, Ginstman et al. reported no clinically significant changes in etonogestrel pharmacokinetics after Roux-en-Y gastric bypass (RYGB), suggesting that oral desogestrel (which is converted to the active metabolite etonogestrel) may be used by women after the procedure; however, the sample size was limited and the lack of sample size calculation limits the ability to draw conclusions. These results need confirmation in larger studies [17].

Mengesha et al. reported in a cross-sectional study that $66 \%$ of 363 reproductive-aged women in the USA after bariatric surgery, recruited through Facebook, used a contraception method postoperatively, of which $27 \%$ were oral contraceptives [18]. In Sweden, Ginstman et al. reported, using a questionnaire study, that in 563 women aged 18-45 years who underwent bariatric surgery, $70.1 \%$ used any contraceptive method, of which $15.5 \%$ were oral contraceptives, and $3 \%$ reported becoming pregnant postoperatively in spite of using contraception. Also, $24.8 \%$ actively stated that they did not receive any contraceptive advice postoperatively [19].
In Belgium, Luyssen et al. found that in 71 women of reproductive age who underwent bariatric surgery, the usage of short-acting hormonal contraceptives decreased from $39.4 \%$ preoperatively to respectively $27.1 \%$ and $14.9 \%$ at 6 and 12 months postoperatively. Menstrual cycle and sexual behavior did not differ before and after surgery [20].

As there is little information known and the possible risk of an unplanned pregnancy after bariatric surgery, the aim of this study was to prospectively assess contraceptive use pre- and postoperatively in women who underwent bariatric surgery in two obesity clinics in The Netherlands. Also, we investigated the recall of contraceptive and pregnancy counseling given during the bariatric care program in these clinics.

\section{Methods}

\section{Study Population}

From October 2017 until August 2018, a web-based questionnaire was sent to all women aged 18-45 years who underwent a bariatric procedure within the last 5 months, in the obesity clinics of Ziekenhuisgroep Twente (ZGT) and Medical Center Leeuwarden (MCL), in The Netherlands. A pregnancy delay of 24 months postoperatively is recommended in these obesity clinics. In the bariatric care program of both clinics, contraceptive and pregnancy counseling is given preoperatively verbally during the information meeting and also in writing in the information brochure. Further counseling may be given by different healthcare providers at any point in the bariatric care program; however, this is not standardized.

Women were identified and enrolled by their bariatric healthcare provider during an information meeting in the bariatric care program who explained the objective of this study and asked for a written informed consent.

Women received the questionnaire using a hyperlink by email and were deidentified in the survey database (SurveyMonkey ${ }^{\circledR}$ ). Those who had not completed the questionnaire within 2 weeks were sent a reminder. Only completed questionnaires were eligible for analysis.

\section{Questionnaire}

The survey focused on contraceptive and pregnancy counseling and contraceptive use pre- and postoperatively in women of reproductive age (18-45 years). This survey was pretested at the outpatient clinic in a focus group. Ten women, who already underwent bariatric surgery, were asked to answer the questions in the questionnaire face to face with one of the researchers, and all comments were noted and used to modify the questionnaire accordingly. 


\section{Objectives}

The primary objective of this study was to assess safe contraceptive use pre- and postoperatively in women who underwent bariatric surgery, defined as the percentage of women who use safe and effective contraception after bariatric surgery according to international guidelines. The use of COCs and POPs was considered unsafe. Long-acting contraceptives (injections, implants, IUDs, and pessaries) were considered safe methods of contraception.

Secondary objectives were to assess the percentage of women who switched from an unsafe contraceptive method to a safe contraceptive method after bariatric surgery and to assess the recall of contraceptive and pregnancy counseling and received information in the bariatric care program. Also, the possible relation between receiving contraceptive counseling and the safe use of contraception postoperatively is investigated.

\section{Statistical Analysis}

SPSS version 25.0 was used to analyze the collected data, using descriptive statistics. Logistic regression was used to analyze the possible relation between use of safe contraception postoperatively and the recall of contraceptive counseling. We determined covariates in the multivariate regression model that we considered potential confounders in the relationship between counseling and postoperative contraceptive use (i.e., age, civil status, education level, obesity clinic, or surgical procedure). A post hoc McNemar-Bowker test was performed to analyze the degree of switching of contraception postoperatively. The level of significance was set at $p<0.05$.

\section{Results}

\section{Study Population}

For this study, 315 women provided a written informed consent and received a questionnaire; 249 women (79.1\%) responded. In total, the results of 230 women were eligible for final analysis. The collected data of 19 women were excluded as they did not meet the inclusion criteria $(N=5)$ or the questionnaire was not filled out completely $(N=3)$. Also, women with a history of surgery for removal of the uterus, ovaries, or oviducts $(N=11)$ were excluded from the analysis as contraceptive use is not relevant for this group. Table 1 shows the baseline characteristics of the study population.

\section{Contraceptive Method Pre- and Postoperatively}

The contraceptive methods used pre- and postoperatively are shown in Fig. 1. Postoperatively, $60 \%$ of women used a safe method of contraception. In this study, $43.7 \%$ of users with
Table 1 Demographic characteristics of study population $(n=230)$

Characteristics

\begin{tabular}{lll}
\hline Age in years, median (IQR) & 35.5 & $(11.0)$ \\
Preoperative weight in kg, mean (SD) & 127.7 & $(17.9)$ \\
Present weight in kg, mean (SD) & 107.3 & $(17.9)$ \\
Preoperative BMI in kg/m ${ }^{2}$, mean (SD) & 43.9 & $(5.5)$ \\
Present BMI in kg/m², mean (SD) & 36.9 & $(5.7)$ \\
Civil status & & \\
No current relationship & 49 & $(21.3 \%)$ \\
Relationship & 181 & $(78.7 \%)$ \\
Education level & & \\
Low (primary school) & 9 & $(3.9 \%)$ \\
Middle (high school) & 169 & $(73.5 \%)$ \\
High (university or other higher & 52 & $(22.6 \%)$ \\
$\quad$ professional education) & & \\
Smoking & 36 & $(15.7 \%)$ \\
Clinic & & \\
ZGT & 166 & $(72.2 \%)$ \\
MCL & 63 & $(27.4 \%)$ \\
Procedure & & \\
RYGB & & \\
OAGB & & \\
Sleeve gastrectomy \\
Time since surgery in months, median (IQR) \\
\hline
\end{tabular}

$B M I$ body mass index, $R Y G B$ Roux-en-Y gastric bypass, $O A G B$ oneanastomosis gastric bypass, $M C L$ Medisch Centrum Leeuwarden, ZGT Ziekenhuisgroep Twente

unsafe contraception preoperatively switched to a safe method of contraception postoperatively $(p<0.0001)$. The use of short-acting unsafe contraceptives COCs and POPs decreased from $30.8 \%$ preoperatively to $16.1 \%$ postoperatively $(p<0.0001)$. Usage of long-acting safe contraceptives increased from 44.7 to $60.0 \%(p<0.0001)$.

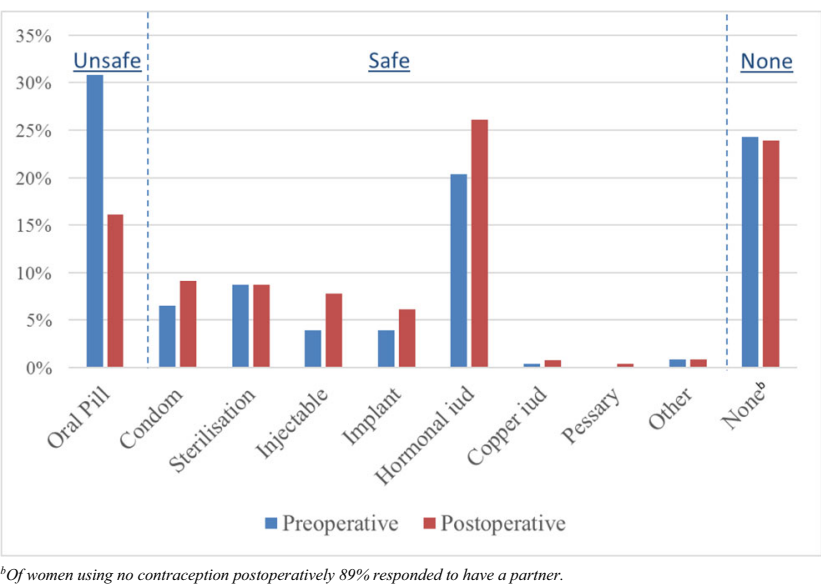

Fig. 1 Contraceptive methods used pre- and postoperatively $(N=230)$. ${ }^{\mathrm{b}}$ Of women using no contraception postoperatively, $89 \%$ responded to have a partner 
Approximately $24 \%$ of women in this study population were not using any method of contraception postoperatively. In this group of women, $89 \%$ reported to be involved in a relationship. Also, four women using unsafe contraception preoperatively switched to using no contraception postoperatively. An overview of contraceptive use classified by safe, unsafe, or no use of contraception pre- and postoperatively respectively is given in Table 2 .

\section{Contraceptive and Pregnancy Counseling}

About $63 \%$ of women confirmed to have received any counseling about contraception, and $83 \%$ about delaying pregnancy in the bariatric care program. Approximately $71 \%$ of women reported to have received information in writing regarding contraception, and $72 \%$ regarding pregnancy in the information meeting preoperatively. In Table 3, we have summarized these results from the questionnaire by healthcare provider and information source.

In this questionnaire, women were asked if they have received counseling about a delay of pregnancy postoperatively and which minimal period of time was advised for this delay. Approximately 53\% of women indicated the recommended delay of 24 months postoperatively as is shown in Table 4.

None of the women was pregnant at the time this questionnaire was taken. Approximately $13 \%$ of women responded that they had the desire to become pregnant within 24 months, and $22 \%$ responded that they respected the recommended delay of 24 months with their desire to become pregnant as is shown in Table 5.

The odds ratio of receiving contraceptive counseling for using safe contraceptive methods compared with no counseling was 2.20 (95\% CI, 1.27-3.79; $p=0.005)$. Women who received contraceptive counseling are more likely to use safe contraceptives. There were no relevant confounders found (age, civil status, education level, obesity clinic, and surgical procedure were checked as possible confounders).
In the overall population, 71 women confirmed to have received contraceptive counseling from one healthcare provider in the bariatric care program, and in this group, $63 \%$ used safe contraception postoperatively.

Eleven women confirmed to have received contraceptive counseling from 4 different healthcare providers, and $73 \%$ of women in this group used a safe method of contraception postoperatively. Despite this difference, we found no statistical significant relation found between receiving contraceptive counseling from more than one healthcare provider and the use of safe contraception postoperatively.

\section{Discussion}

This study in two obesity clinics in The Netherlands shows that $60 \%$ of women who underwent bariatric surgery are using a safe method of contraception postoperatively according to international guidelines. About $24 \%$ of women who underwent bariatric surgery responded not to use any method of contraception postoperatively. As the questionnaire did not include questions regarding the status of sexual activity, we could not distinguish the actual need for contraception in this population. However, it is to be noted that in this group of women, $89 \%$ reported to be involved in a relationship, though we have no information whether these relationships were heterosexual relationships and sexual active relationships.

Approximately $63 \%$ of women could confirm to have received counseling or information about contraception after bariatric surgery. These results suggest that the quality of counseling and prescribing of contraception in women who undergo bariatric surgery needs improvements.

In this study, we observed a decrease in use of oral contraceptives postoperatively; however, still $16.1 \%$ of women continued in using this unsafe method of contraception, according to international guidelines. Further pharmacokinetic studies

Table 2 Overview of contraceptive use classified as safe, unsafe, and no use of contraception pre- and postoperatively $(N=230)$

\begin{tabular}{|c|c|c|c|c|c|c|}
\hline & & & \multicolumn{4}{|l|}{ Postoperative } \\
\hline & & & Safe contraception & Unsafe contraception & No contraception & Total \\
\hline \multirow[t]{8}{*}{ Preoperative } & \multirow[t]{2}{*}{ Safe contraception } & $N$ & 103 & 0 & 0 & 103 \\
\hline & & $\%$ & $100.0 \%$ & $0.0 \%$ & $0.0 \%$ & $100.0 \%$ \\
\hline & \multirow[t]{2}{*}{ Unsafe contraception } & $N$ & 31 & 36 & 4 & 71 \\
\hline & & $\%$ & $43.7 \%$ & $50.7 \%$ & $5.6 \%$ & $100.0 \%$ \\
\hline & \multirow[t]{2}{*}{ No contraception } & $N$ & 4 & 1 & 51 & 56 \\
\hline & & $\%$ & $7.1 \%$ & $1.8 \%$ & $91.1 \%$ & $100.0 \%$ \\
\hline & \multirow[t]{2}{*}{ Total } & $N$ & 138 & 37 & 55 & 230 \\
\hline & & $\%$ & $60.0 \%$ & $16.1 \%$ & $23.9 \%$ & $100.0 \%$ \\
\hline
\end{tabular}


Table 3 Overview of survey responses for receiving pre- and postoperative counseling in contraception and/or pregnancy $(N=$ 230)

\begin{tabular}{|c|c|c|c|c|}
\hline \multirow[t]{2}{*}{ Received counseling } & \multicolumn{2}{|c|}{ Contraceptive information } & \multicolumn{2}{|c|}{ Pregnancy information } \\
\hline & $N$ & $(\%)$ & $N$ & $(\%)$ \\
\hline Yes & 144 & $(62.6)$ & 192 & $(83.5)$ \\
\hline No & 86 & $(37.4)$ & 38 & $(16.5)$ \\
\hline \multicolumn{5}{|c|}{ If yes, when and by which healthcare provider counseling was given ${ }^{\mathrm{a}}$ : } \\
\hline \multicolumn{5}{|l|}{ Preoperative by: } \\
\hline Surgeon & 50 & $(34.7)$ & 78 & $(40.6)$ \\
\hline Internist & 22 & $(15.3)$ & 33 & $(17.2)$ \\
\hline Nurse specialist obesity & 58 & $(40.3)$ & 88 & $(45.8)$ \\
\hline Outpatient obesity nurse & 72 & $(50.0)$ & 105 & $(54.7)$ \\
\hline General practitioner & 12 & $(8.3)$ & 17 & $(8.9)$ \\
\hline \multicolumn{5}{|l|}{ Postoperative by: } \\
\hline Surgeon & 5 & $(3.5)$ & 8 & $(4.2)$ \\
\hline Internist & 2 & (1.4) & 5 & (2.6) \\
\hline Nurse specialist obesity & 14 & $(9.7)$ & 24 & $(12.5)$ \\
\hline Outpatient obesity nurse & 22 & $(15.3)$ & 28 & $(14.6)$ \\
\hline General practitioner & 3 & $(2.1)$ & 3 & (1.6) \\
\hline Other healthcare providers & 6 & $(4.2)$ & 10 & $(5.2)$ \\
\hline \multicolumn{5}{|l|}{ Information sources: } \\
\hline Information meeting & 102 & $(70.8)$ & 139 & $(72.4)$ \\
\hline Information map & 47 & $(32.6)$ & 67 & $(34.9)$ \\
\hline Internet & 43 & $(29.9)$ & 43 & $(22.4)$ \\
\hline Conversation & 51 & $(35.4)$ & 104 & $(54.2)$ \\
\hline Group meeting & 50 & $(34.7)$ & 57 & $(29.7)$ \\
\hline Other & 1 & $(0.7)$ & 1 & $(0.5)$ \\
\hline
\end{tabular}

${ }^{\text {a }}$ Participants could select $>1$ choice investigating whether bariatric surgery affects the pharmacokinetics of oral contraceptives are required to set this in more perspective.

Our results regarding the use of contraception are in line with the Swedish study done by Ginstman et al. $(N=563)$ in which $15.5 \%$ were using oral contraceptives postoperatively and $29.9 \%$ did not use any method of contraception [19]. In the study from the USA by Mengesha et al. $(N=363)$, these percentages were higher, respectively $27 \%$ oral contraceptives and $34 \%$ no contraception postoperatively [18].

Table 4 Overview of survey responses for delaying pregnancy $(N=$ 230)

\begin{tabular}{lll}
\hline Advised delay for pregnancy & $N$ & $(\%)$ \\
\hline 12 months & 50 & $(21.7 \%)$ \\
18 months & 15 & $(6.5 \%)$ \\
24 months & 121 & $(52.6 \%)$ \\
Other (with remarks) & 6 & $(3.1 \%)$ \\
- Stabilized weight & & \\
\hline
\end{tabular}

We also found that adequate contraceptive counseling in the bariatric care program is important as this significantly improves the use of safe contraception postoperatively. However, we cannot exclude any influence of other contributing factors which were not investigated in this study.

As only $62.6 \%$ in our study could confirm to have received contraceptive counseling and only $52.6 \%$ could indicate the recommended pregnancy delay of 24 months postoperative, it is worth thinking about how to improve this.

Table 5 Overview of survey responses regarding the desire to become pregnant $(N=230)$

\begin{tabular}{lll}
\hline Desire to become pregnant & $N$ & $(\%)$ \\
\hline No & 149 & $(64.8 \%)$ \\
Yes, but not within 24 months & 50 & $(21.7 \%)$ \\
Yes, within 24 months & 27 & $(11.7 \%)$ \\
Yes, within 12 months & 4 & $(1.7 \%)$ \\
Now pregnant & 0 & $(0.0 \%)$ \\
\hline
\end{tabular}


It is notable that in our study, counseling was predominantly recalled as given preoperatively and by the obesity nurse and nurse specialist in the bariatric care program. Perhaps postoperatively counseling is more effective as this is the setting when it is most relevant and at this point after the operation, women may be more inclined to follow up on this. Counseling by more than one healthcare provider may decrease the risk of using unsafe contraception postoperatively; however, this was not statistically significant.

It may also be worthwhile to include general practitioners and pharmacists in the outpatient setting in monitoring whether women who underwent bariatric surgery are using safe contraception as they usually prescribe and dispense this contraception. For this reason, it is important that obesity clinics actively inform these healthcare providers about the bariatric surgery after discharge from the hospital. Furthermore, bariatric surgery should be registered as a condition in the electronic medical record of all healthcare providers for these women with adequate signaling when contraindicated drugs, like oral contraception, are prescribed or dispensed.

\section{Strengths and Limitations}

The results of this questionnaire study regarding contraceptive and pregnancy counseling by whom and when, may have been affected by recollection bias as the time between participating in this study and the moment of counseling may have been too long for some women for remembering this always correctly. This study was also limited as we did not include questions in the questionnaire regarding the status of sexual activity as this may be a reason for not using contraception. Final conclusions for this group must therefore be drawn with caution.

The high response rate is the strength of this study. Therefore, the results represent a good reflection of this bariatric surgery population in the two obesity clinics in The Netherlands investigated. The differentiation by whom and when counseling was given, is informative and strengthens this study, which we have used to formulate specific recommendations for improvements in counseling regarding contraception after bariatric surgery.

\section{Conclusions}

In this questionnaire study, we found that a substantial part of women who underwent bariatric surgery is using potential unsafe or no contraception. In this way, they are not only at risk for an unwanted and unplanned pregnancy, but because of the weight loss in the early months after bariatric surgery also at risk for a pregnancy with adverse effects and complications. Contraceptive and pregnancy counseling is suboptimal, and improvements are necessary as we have demonstrated that this is correlated with the use of safe contraception postoperatively. We suggest implementing more counseling and monitoring in the postoperative and in the outpatient setting. Adequate signaling in the electronic medical information systems for contraindicated drugs like oral contraception after bariatric surgery could be helpful for better monitoring and guidance.

Acknowledgments We would like to thank all participants in this study and co-workers of the obesity clinics in Hengelo and Leeuwarden, who participated in this study. We thank F.R. Pierik, Department of Pharmacy, University of Groningen, for his help in collecting data from the surveys.

\section{Compliance with Ethical Standards}

Ethical Approval All procedures performed in studies involving human participants were in accordance with the ethical standards of the institutional and/or national research committee and with the 1964 Helsinki Declaration and its later amendments or comparable ethical standards.

Informed Consent Informed consent was obtained from all individual participants included in the study.

Conflict of Interest The authors declare that they have no conflict of interest.

Open Access This article is distributed under the terms of the Creative Commons Attribution 4.0 International License (http:// creativecommons.org/licenses/by/4.0/), which permits unrestricted use, distribution, and reproduction in any medium, provided you give appropriate credit to the original author(s) and the source, provide a link to the Creative Commons license, and indicate if changes were made.

\section{References}

1. Trus TL, Pope GD, Finlayson SRG. National trends in utilization and outcomes of bariatric surgery. Surg Endosc. 2005;19:616-20.

2. Pratt GM, Learn CA, Hughes GD, et al. Demographics and outcomes at American Society for Metabolic and Bariatric Surgery Centers of Excellence. Surg Endosc. 2009;23:795-9.

3. Paulen ME, Zapata LB, Cansino C, et al. Contraceptive use among women with a history of bariatric surgery: a systematic review. Contraception. 2010;82:86-94.

4. Burke A, Jamshidi R, Eaton LSK. Contraceptive use and unplanned pregnancy after bariatric surgery: results from the reproductive outcomes after bariatric surgery survey. Contraception. 2008;78:16795 .

5. Wittgrove AC, Jester L, Wittgrove P, et al. Pregnancy following gastric bypass for morbid obesity. Obes Surg. 1998;8:461-4.

6. Patel JA, Patel NA, Thomas RL, et al. Pregnancy outcomes after laparoscopic Roux-en-Y gastric bypass. Surg Obes Relat Dis. 2008;4:39-45.

7. Dao T, Kuhn J, Ehmer D, et al. Pregnancy outcomes after gastricbypass surgery. Am J Surg. 2006;92:762-6.

8. Kjær MM, Nilas L. Timing of pregnancy after gastric bypass - a national register-based cohort study. Obes Surg. 2013;23:1281-5.

9. Lesko J, Peaceman A. Pregnancy outcomes in women after bariatric surgery compared with obese and morbidly obese controls. Obstet Gynecol. 2012;119:547-54.

10. Josefsson A, Bladh $\mathrm{M}$, Wiréhn $\mathrm{AB}$, et al. Risk for congenital malformations in offspring of women who have undergone 
bariatric surgery. A national cohort. BJOG An Int J Obstet Gynaecol. 2013;120:1477-82.

11. Johansson K, Cnattingius S, Näslund I, et al. Outcomes of pregnancy after bariatric surgery. N Engl J Med. 2015;372:814-24.

12. Merhi ZO. Impact of bariatric surgery on female reproduction. Fertil Steril. 2009;92:1501-8.

13. Centers for Disease Control and Prevention. U.S. medical eligibility criteria for contraceptive use, 2016. Morb Mortal Wkly Rep. 2016;65:1-106.

14. Merki-Feld GS, Skouby S, Serfaty D, et al. European Society of Contraception statement on contraception in obese women. Eur J Contracept Reprod Health Care. 2015;20:19-28.

15. Andersen AN, Lebech PE, Sørensen TI, et al. Sex hormone levels and intestinal absorption of estradiol and D-norgestrel in women following bypass surgery for morbid obesity. Int J Obes. 1982;6: 91-6.

16. Victor A, Odlind VKJ. Oral contraceptive absorption and sex hormone binding globulins in obese women: effects of jejunoileal bypass. Gastroenterol Clin N Am. 1987;16:483-91.
17. Ginstman C, Frisk J, Carlsson B, et al. Plasma concentrations of etonogestrel in women using oral desogestrel before and after Roux-en-Y gastric bypass surgery: a pharmacokinetic study. BJOG An Int J Obstet Gynaecol. 2019;126:486-92.

18. Mengesha BM, Carter JT, Dehlendorf CE, et al. Perioperative pregnancy interval, contraceptive counseling experiences, and contraceptive use in women undergoing bariatric surgery. Am J Obstet Gynecol. 2018;219:81.e1-9.

19. Ginstman C, Frisk J, Ottosson J, et al. Contraceptive use before and after gastric bypass: a questionnaire study. Obes Surg. 2015;25: 2066-70.

20. Luyssen J, Jans G, Bogaerts A, et al. Contraception, menstruation, and sexuality after bariatric surgery: a prospective cohort study. Obes Surg. 2018;28:1385-93.

Publisher's Note Springer Nature remains neutral with regard to jurisdictional claims in published maps and institutional affiliations. 